\title{
Heterogeneous Wireless System Testbed for Remote Image Processing in Automated Vehicles
}

\author{
Cristian Roman*, Michael Sapienza ${ }^{\dagger}$, Peter Ball*, Shumao Ou*, Fabio Cuzzolin* and Philip H.S. Torr ${ }^{\dagger}$ \\ * Department of Computing and Communication Technologies, Oxford Brookes University, Oxford, UK \\ Email: \{croman.2012, pball, sou, fabio.cuzzolin\}@brookes.ac.uk \\ $\dagger$ Department of Engineering Science, University of Oxford, Oxford, UK \\ Email: \{michael.sapienza, philip.torr\}@eng.ox.ac.uk
}

\begin{abstract}
Automated vehicles will carry computing and communication platforms, and will have enhanced sensing capabilities. Safety around people along with obstacle detection and avoidance systems are key to their success. In controlled environments, automated vehicles can benefit from a remote processing approach to reduce cost and accelerate deployment on larger scales. In this paper we present a section of our intelligent transport systems testbed which evaluates the remote image processing approach with a novel heterogeneous wireless communication system. Hardware implementation is carried out for an experimental evaluation and comparison with the simulation results.
\end{abstract}

\section{INTRODUCTION}

Autonomous or robot vehicles will be able to navigate and drive themselves. They need to analyse the road automatically to identify road markings, street furniture, and other road users, and to adapt to the road surface. And, they need to be safe around people. The scope of automated vehicles or partly automated vehicles goes beyond the typical city/transport vehicle scenario. Partially automated vehicles could be used in more controlled environments, such as hospitals/airports and have less intelligent autonomous capabilities in order to reduce cost and have a faster implementation on a large scale. Even though the implementation of computer vision algorithms in their more elementary aspects is now possible on a low cost desktop computer workstation, advanced image processing applications still require the use of dedicated high performance peripheral processors. In that sense, the advancement made in wireless communications can be used to distribute processing among specialized nodes or to a centralized server. Concepts have also changed from completely autonomous vehicles where a unit acts and takes decisions independently to a more automated and connected approach. The vehicle can still make decisions based only on its sensors but gathering and having information from multiple surrounding sources helps in the decision making, enhancing transportation safety and efficiency, in addition to infotainment provisioning.

Rather than implementing an expensive, computational intensive algorithm on each of the vehicles, a central server can process all the information captured by the sensors and the commands are to be sent back to the car. Cloud computing

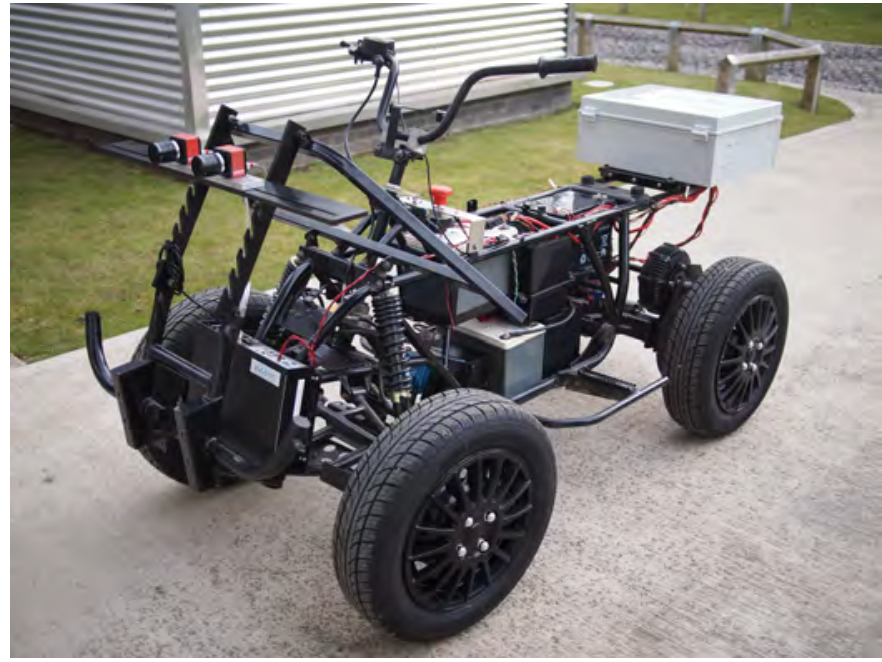

Fig. 1: The Brookes Intelligent Transport Systems Off-road all-terrain test vehicle intended to be like a 'pack mule' that can follow a person and avoid obstacles

with remote image processing can be a solution. These cars can thus be controlled as a fleet.

Nevertheless, no single wireless technology is yet capable of answering to all the communication requirements of such a new versatile system. A new user based model using the existing wireless standards, Multiple Interface Scheduling System (MISS), presented in Section III-A capable of responding to this demand has been developed, simulated and tested. The objective of this work is to implement the proposed scheduling system on hardware and compare the simulated results (Section IV) with the practical measurements using the the Oxford Brookes Intelligent Transport Systems (OBU-ITS) test bed, described in Section III Initial remote image processing tests have been performed with traversability segmentation computer vision algorithms (Section III-B) with a single Radio Access Technology (RAT). This paper also aims to show the feasibility of obstacle avoidance using low-cost equipment in automated vehicles using a remote processing approach in a real-time environment. 


\section{ThE OXFORD BROOKES INTELLIGENT TRANSPORT Systems TEST BED}

The OBU-ITS platform, as described in [1], is a University constructed electric powered autonomous off-road all-terrain vehicle based on a full-size quadbike (Fig. 1). The vehicle is intended to be like a 'pack mule' that has its own sense of situational awareness around groups of people, and can follow simple commands such as 'follow me', 'park over there', and 'unload yourself from the van'. A variety of projects are contributing to the design and construction of this vehicle:

\section{A. Intelligent motor control for electric vehicles}

The control system needs to use the dynamic response of the vehicle to the motor torque to evaluate the tractive qualities of the terrain it is moving over, to adjust its operation so that traction is maintained, and to ensure that the motors are used at the most efficient torque and speed [2].

\section{B. Road scene understanding}

Abundant access to street level imagery was obtained from in-car cameras. The application is to automatically inspect the street level imagery to assay road signs, street furniture, and other roadside assets. The approach combines appearance information with structure-from-motion features to understand the road scene.

\section{Visual guidance and navigation}

Developing real-time visual guidance software and hardware that uses colour stereo image sequences plus information from inertial sensors. More details of the current implemented algorithm is given in Section (III-B).

\section{Heterogeneous Wireless Networks}

The communication system uses heterogeneous wireless access technology to accommodate different coverage areas. The objectives are both to reduce delay and increase the quality of the video images in order for the vision algorithm to have the best images possible. To this end a scheduling algorithm for an adaptive heterogeneous wireless communication has been devised to have 1) automatic wireless access interface selection; 2) intelligent bandwidth aggregation and allocation; 3) seamless QoS support; and 4) context-aware packet scheduling. The term heterogeneous means that a combination of different wireless technologies may be used: cellular technologies, such as 4G-LTE (Long Term Evolution), Wi-Fi technologies (802.11n 2.4GHz/5GHz) and WAVE (802.11p) are considered in this case. A full detail of all the tested technologies is presented in Section III-A.

\section{BACKGROUND}

The main advantage of remote processing is the reduction in cost and a better utilization of all the available resources. The technique is not specific to ITS. Remote medical diagnosis has been used in the past decade [3]. Earth science and social science researchers also use analysis of remotely sensed data by computer to benefit their decision-making capability

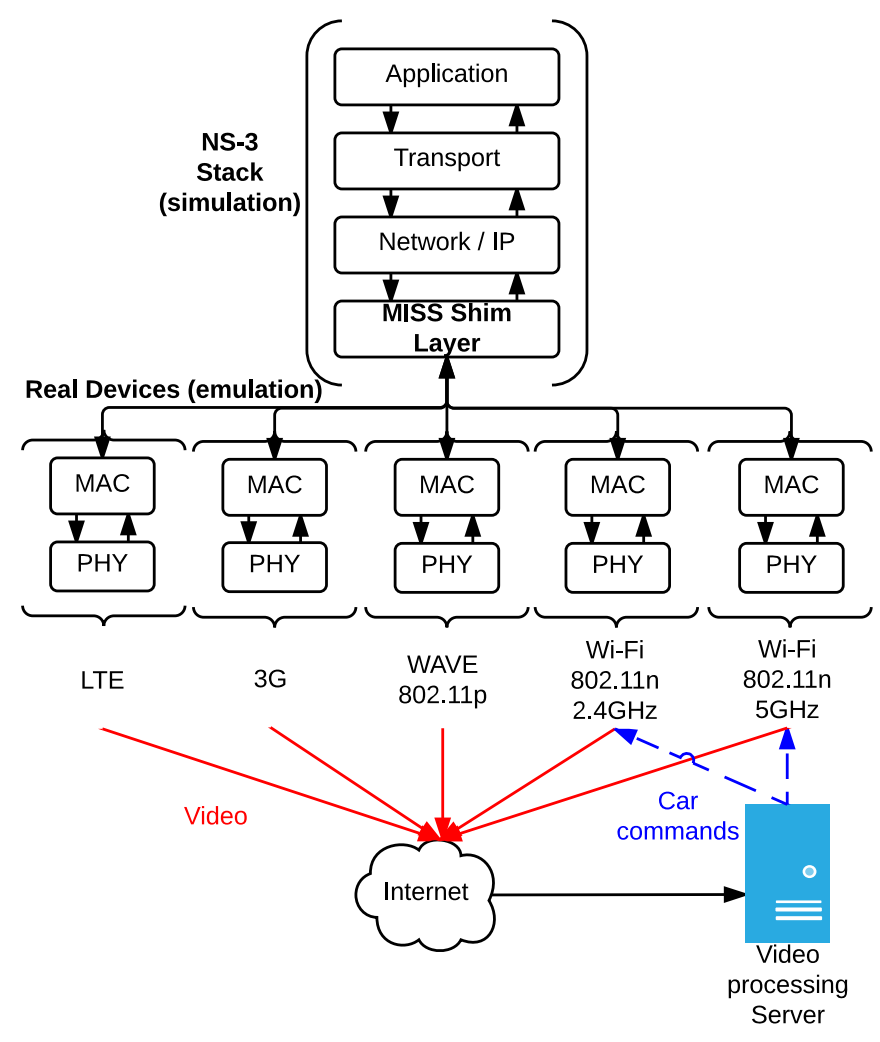

Fig. 2: Conceptual model of the common layers from the IP Layer upwards along with the Shim Layer's interaction with the emulated layers. The data is uploaded via all interfaces and received only via Wi-Fi.

since 1986 [4]. The large amount of information collected and processed leads to massive remote processing [5] which brings another set of challenges related to prioritization of processing tasks and allocation of resources. These issues are out of the scope of this paper and have been left for a further study.

In this section the wireless communication and computer vision algorithm of the OBU-ITS are described in more detail.

\section{A. Wireless Communication}

Access to multiple technologies can improve the performance of Vehicle to Vehicle (V2V) and Vehicle to Infrastructure (V2I) communications. Rather than finding the best alternative amongst different technologies, our proposed solution, MISS [6], uses simultaneously all the available networks, without modification of the wireless standards, that can meet multiple criteria. The packet scheduler dynamically selects the most suitable wireless technology in a given space and time or may use the technologies jointly to maximize the throughput or improve the coverage and reliability.

The system is located at the user side in a shim layer between the MAC and the Network/IP layer. MISS classifies the received messages in five different queues (safety critical, video, voice, best-effort and background), prioritizes safety messages, and/or video, and selects the best interface for a packet based on different profiles. The advantage of our 


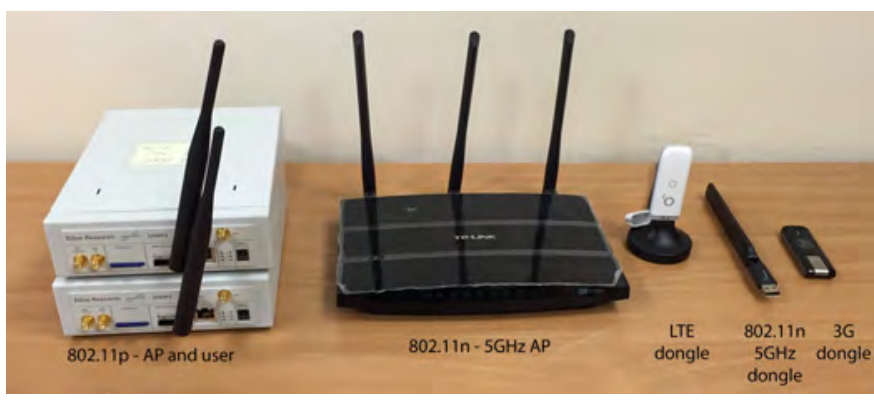

Fig. 3: Interfaces to be used on the OBU-ITS testbed (dongles)

approach is the use of a single IP layer and thus the use of a single IP address per vehicle. The scheduling algorithm could encompass any of the wireless standards based on IP being developed either in the car industry or the Telecom industry. The possibility of extending the communication to an ad-hoc network with one or more miniature cars that take instructions through the quad bike is also considered.

The conceptual model of the shim layer and the communication flow can be observed in Fig. 2. The data is being uploaded via all the available interfaces based on the scoring system in the MISS algorithm [7]. It is to be noted that only two links are used for the return path.

The implementation consists of the emulation mode of NS-3 (Network Simulator $3[8]$ ) to interface with all the mentioned RATs. The detail of the wireless equipment used is listed below and the hardware can be seen in Fig. 3

1) WAVE: We use the work made available by [9] to have a full 802.11p SDR based 802.11 Transceiver using GNU Radio. The GNU Radio [10] is to be interfaced with NS-3.

2) 3G/LTE: One dongle for each technology $3 G / 4 G(L T E)$.

3) WiFi: The internal network card of the ITS laptop is used (Broadband BCM43224) for the 802.11n 2.4GHz. The $802.11 \mathrm{n} 5 \mathrm{GHz}$ is tested with an external antenna.

All the interfaces are connected to a laptop currently placed on the quad bike, but other smaller devices could be considered in the future. We assume there is no constraint on the power consumption. Vehicles can provide more resources than other types of mobile networks such as large batteries, antennas, and processing power. Therefore, conserving such resources in vehicular networks is not a major concern.

\section{B. Computer Vision}

In order to demonstrate the capability of remote image processing for the OBU-ITS robotic platform, we used a computer vision algorithm which automatically guides the robot away from oncoming obstacles. A camera was placed at the front of the OBU-ITS platform and images were then transmitted to a server. The vision algorithm takes as input an RGB image and outputs a speed and steering angle to re-direct the autonomous platform. After performing semi-supervised image segmentation into traversable and non-traversable regions (Fig. 5), the vision algorithm estimates the distance to the nearest obstacles using trigonometric identities (Fig. 4).

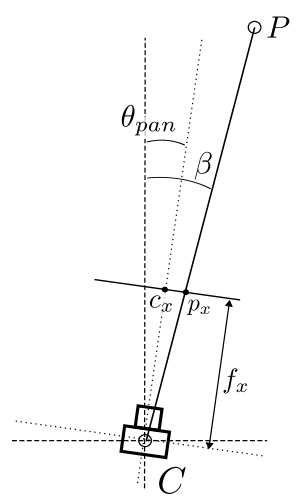

$\beta=\theta_{\text {pan }}+\arctan \frac{p_{x}-c_{x}}{f_{x}}$

Orientation

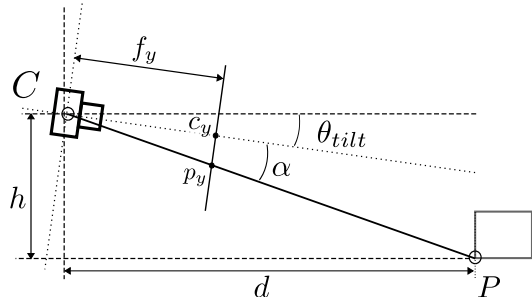

$$
\alpha=\arctan \frac{c_{y}-p_{y}}{f_{y}}
$$$$
d=h \cot \left(\theta_{t i l t}+\alpha\right)
$$

Distance
Fig. 4: Assuming that the ground is planar, consider a point $\mathrm{P}$ in the world and its corresponding projection onto the image plane. With knowledge of the camera focal length and centre, the orientation and depth of an obstacle can be estimated using trigonometric identities.

Finally the distance estimates are analysed and the largest obstacle free areas beyond a predefined distance are identified as possible movement directions [11]. The code for the realtime autonomous guidance algorithm is available online [12]. We are currently exploring the possibility of using more robust 3D semantic segmentation [13].

Most computer vision algorithms do not work with high resolution images in real-time as it is a challenging requirement, especially for mobile and embedded computing architectures [14]. Often, it is possible to trade off quality for speed. A resolution image of $640 \times 480$ or higher, with 30 frames per second, is necessary for fine-grained boundaries. If we assume such a resolution, a required 27.6 MBps data rate is required. If we assume a $1024 \times 768$ resolution, $141.25 \mathrm{Mbps}$ is needed. Heterogeneous systems become thus a necessity as none of the current wireless technologies can provide such data rates in non-ideal environments. Additionally, in vehicular networks, the links tend to be far less predictable than in a more static environment. This characteristic prompts the development of opportunistic higher layers that should take advantage of a good link while it lasts without counting on its longevity.
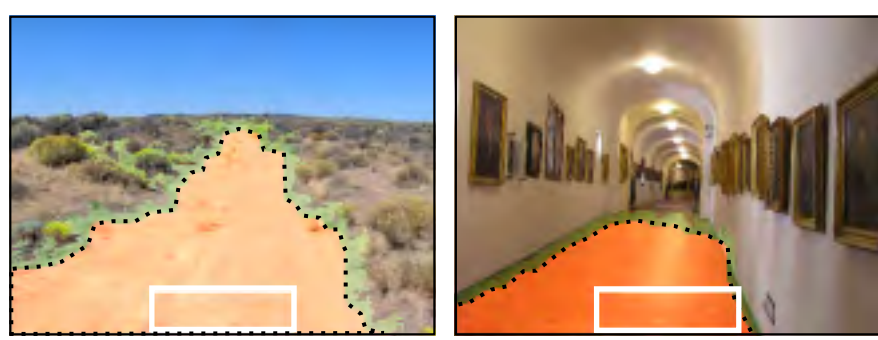

Fig. 5: Examples of image segmentation into traversable and non-traversable regions in both outdoor and indoor environments. 


\section{Simulation and Initial Test Bed Results}

In this section the network simulation results with a video evaluation framework, EvalVid [15] are presented. Due to its modular structure, EvalVid makes it possible to exchange at users discretion both the underlying transmission system as well as the codecs, so it is applicable to any kind of coding scheme, and can be used both in real experimental set-ups and simulation experiments. The initial test bed results are also presented, along with a description of the testing environment around the university campus.

\section{A. Simulation Results}

The simulations were performed using NS-3 in a scenario with a single user, single RAT available $(802.11 \mathrm{n} 5 \mathrm{GHz}$ with $27 \mathrm{Mbps}$ bandwidth) and no interference, similar to the ITSOBU testbed. The video quality transmission (Fig. 6 and Fig. 7) were evaluated with the 'Highway' video (2000 frames) from the EvalVid framework. The full results and complete description are published in [16] [6] [7].

In a saturated environment or where the available resources can not respond to the video demand the algorithm applies the 'optimized video' profile. The MISS algorithm distinguishes between the different video frame types and places the most important frames (e.g. I Frames for MPEG-4) in the safety queue. The other frames remain placed in the designated video queue. In a non saturated environment, all the packets are transmitted using the standard MISS system scheduling scheme. The results are presented in Fig. 6 - the data rates under $30 \mathrm{Mbps}$ (no saturation) are not plotted as they have a $100 \%$ delivery rate and a MOS close to ideal. It can be observed that when I Frames are tagged as safety packets, even if the received Number of Frames (NoF) decrease with an increasing load, the MOS is relatively constant compared to a standard MISS approach. For 137 extra frames transmitted (6.85\% of total frames) there is a quality improvement of $54 \%$ (1.4 compared to 2.77). On the contrary when there is no differentiation between the video frames, the MOS degrades with the number of received frames. It can also be observed that the number of received frames is constant after a 80 Mbps load, as a result of the applied CoDel queue in the shim layer. The CoDel queue [17] eliminates the expired saturation packets from the queue, thus reducing the queue time. Likewise, the simulation results in Fig. 7] show that MISS improves the Mean Opinion Score (MOS) of transmitted video in saturated mobile environments compared to classic switched scheme approaches - use of single RAT with no prioritization of packets. Even if the available bandwidth does not meet the demand of the video, the scheduling algorithm preserves a good video quality level necessary for the image processing algorithm.

\section{B. Initial Test Bed Results}

The test environment can be seen in Fig. 8 as well as the received signal strength on the roads and fields accessible by the quad bike around the campus. The received signal strength measurements have been performed using Wi-Fi Explorer on

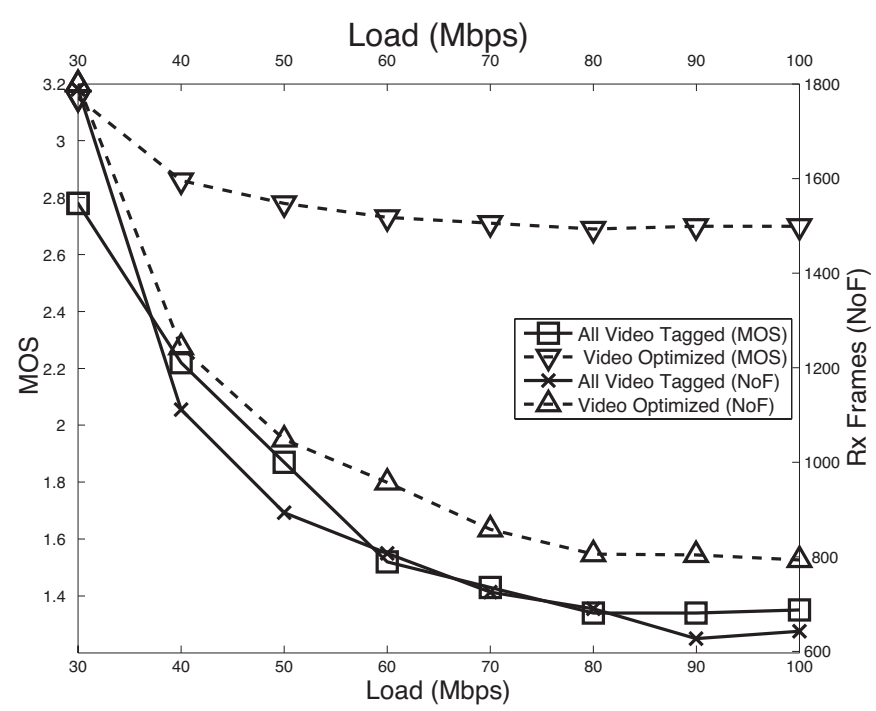

Fig. 6: Comparison between MOS and Number of Received Frames with and without MISS Video Optimization enabled with a single RAT in a saturated environment (Simulation)

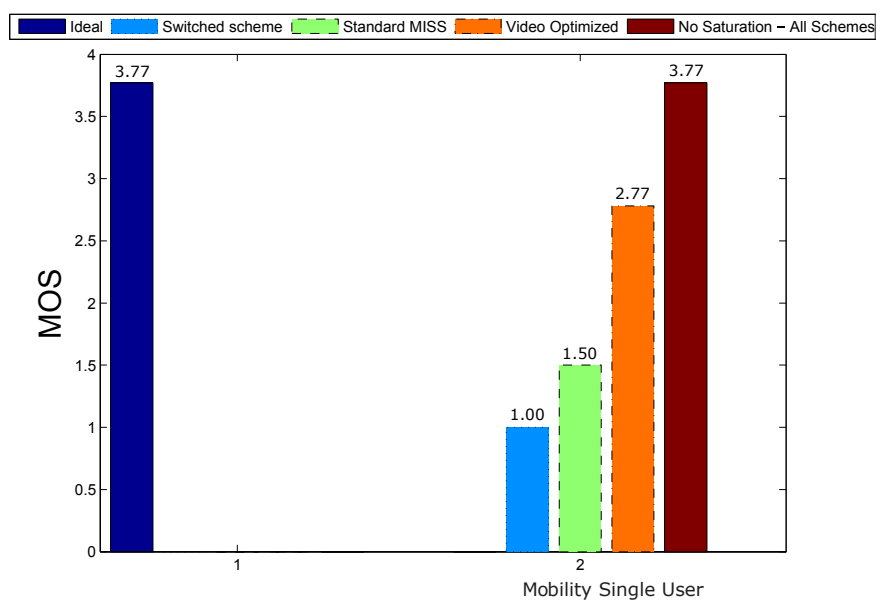

Fig. 7: Summary of received video MOS Scores with a single RAT in a saturated environment (Simulation)

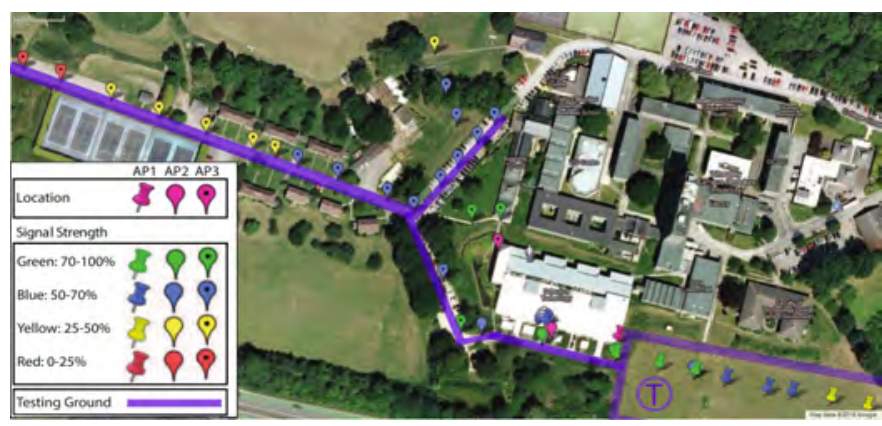

Fig. 8: Map of Testing Environment on Wheatley Campus with Received Signal Strength measurements based on a single RAT: 802.11 n $2.4 \mathrm{GHz}$ 


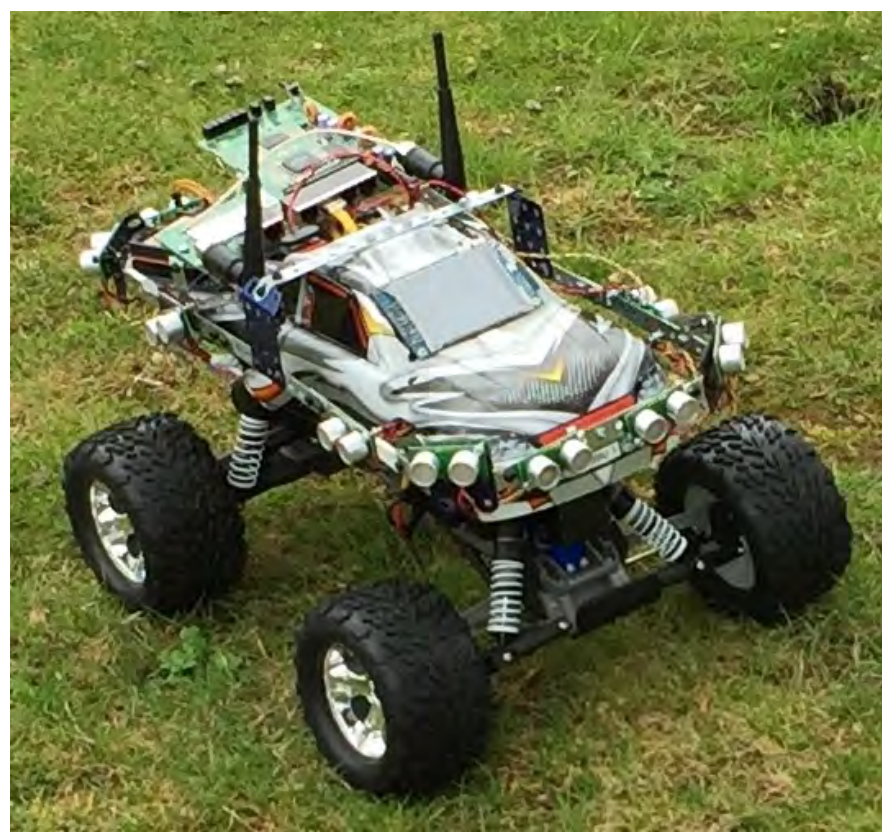

Fig. 9: Brookes Rover

a sunny, windy day (ambient temperature 2C). The receiver, a laptop with AirPort Extreme firmware version Broadcom BCM43xx 1.0 was always oriented towards the closest line of sight access point. Three $802.11 \mathrm{n} 2.4 \mathrm{GHz}$ AP are available, placed around the $\mathrm{R}$ building. $802.11 \mathrm{n} 5 \mathrm{GHz}$ AP are to be mounted at the same location as the $2.4 \mathrm{GHz}$ APs.

Initial tests have been performed on the isolated field, marked with a (T) on Fig. 8 with the quad bike speed limited at $5 \mathrm{~km} / \mathrm{h}$. The quad bike avoided the cone obstacles successfully. On a single thread the computer vision algorithm took approximately $5 \mathrm{~ms}$ to run on a single image of size $160 \times 120$. In the scenario with a single Wi-Fi interface and no optimization of the standard Wi-Fi algorithms, the round trip for the control message to reach the quad bike was around $400 \mathrm{~ms}$, with values up to $800 \mathrm{~ms}$. The value needs to be considerably lower for an efficient obstacle avoidance detection at higher speeds. Improvement can be achieved on the encoding and compression of the video on the transmitter side as well as the optimization of the network.

For development purposes, a miniature version of the quadbike has also been developed, named the 'Brookes Rover' (Fig 9). It is equipped with a Raspberry $\mathrm{Pi}$ and camera for video streaming, Arduino boards for the ultrasonic sensors and a wireless $2.4 \mathrm{GHz}$ communication interface. The ultrasonic sensors are used as a backup solution in case the computer vision algorithms fail to identify an obstacle. For more information the reader is also invited to see the available online project video [18].

\section{CONCLUSION}

An Intelligent Transport Systems test bed is under development to assess remote image processing capabilities via heterogeneous wireless systems. Tests have been performed with a single wireless technology and the feasibility of obstacle avoidance using low cost equipment with a remote image processing approach in a real time environment has been shown. Work will be undertaken on the hardware implementation of the proposed algorithm with multiple wireless interfaces to compare with the simulation results.

\section{REFERENCES}

[1] "William clocksin - founding director of the oxford brookes university intelligent transport systems programme - webpage," http://www. clocksin.com/page5/page10/page10.html accessed: 2014-04-29.

[2] M. Cecotti, J. Larminie, and B. Azzopardi, "Estimation of slip ratio and road characteristics by adding perturbation to the input torque," in Vehicular Electronics and Safety (ICVES), 2012 IEEE International Conference on. IEEE, 2012, pp. 31-36.

[3] F. P. DiFilippo, A. Sivashankaran, S. M. Behm, and B. A. Cottrill, "Remote medical image analysis," Dec. 7 2004, uS Patent 6,829,378.

[4] J. R. Jensen, "Introductory digital image processing: a remote sensing perspective," Univ. of South Carolina, Columbus, Tech. Rep., 1986.

[5] W. Zhang, L. Wang, D. Liu, W. Song, Y. Ma, P. Liu, and D. Chen, "Towards building a multi-datacenter infrastructure for massive remote sensing image processing," Concurrency and Computation: Practice and Experience, vol. 25, no. 12, pp. 1798-1812, 2013.

[6] C. Roman, P. Ball, and S. Ou, "A shim layer for heterogeneous wireless communications scheduling in intelligent transport systems," in Symposium on Computers and Communications(ISCC), 2015 20th Symposium IEEE. IEEE, 2015.

[7] — "Performance evaluation of dynamic scheduler in multiple queue shim layer for heterogeneous vehicular communications," in IEEE International Workshop on Advances in Software Defined Radio Access Networks and Context-aware Cognitive Networks (IEEE SDRANCAN) in conjunction with IEEE GLOBECOM, 2015. IEEE, 2015.

[8] “Ns-3," https://www.nsnam.org/. accessed: 2016-03-09.

[9] B. Bloessl, M. Segata, C. Sommer, and F. Dressler, "Towards an Open Source IEEE 802.11p Stack: A Full SDR-based Transceiver in GNURadio," in 5th IEEE Vehicular Networking Conference (VNC 2013). Boston, MA: IEEE, December 2013, pp. 143-149.

[10] G. Radio, "The gnu software radio," Available from World Wide Web: https://gnuradio. org, 2007.

[11] M. Sapienza and K. P. Camilleri, "A generative traversability model for monocular robot self-guidance." in ICINCO (2), 2012, pp. 177-184.

[12] "Michael sapienza webpage - downloads," https://sites.google.com/site/ mikesapi/downloads accessed: 2016-02-22.

[13] S. Sengupta, E. Greveson, A. Shahrokni, and P. H. Torr, "Urban 3d semantic modelling using stereo vision," Proceedings of IEEE International Conference on Robotics and Automation (ICRA), 2013.

[14] K. Pulli, A. Baksheev, K. Kornyakov, and V. Eruhimov, "Real-time computer vision with opencv," Communications of the ACM, vol. 55, no. 6, pp. 61-69, 2012.

[15] J. Klaue, B. Rathke, and A. Wolisz, "Evalvid-a framework for video transmission and quality evaluation," in Computer performance evaluation. Modelling techniques and tools. Springer, 2003, pp. 255-272.

[16] C. Roman, P. Ball, and S. Ou, "Multiple interface scheduling system for heterogeneous wireless vehicular networks: Description and evaluation," Special Issue of Software Defined Radio and Network of the EAI Transactions on Wireless Spectrum, to be published 2016.

[17] K. Nichols and V. Jacobson, "Controlling queue delay," Communications of the ACM, vol. 55, no. 7, pp. 42-50, 2012

[18] “Oxford brookes its description video," https://youtu.be/Z5gHR1945jA accessed: 2016-03-01. 